\title{
Rapid Detection of Tebuconazole Based on Aptasensor and Aggregation of Silver Nanoparticles
}

\author{
Phuoc Long Truong $\mathbb{D}^{1,2}$ Vo Thi Cam Duyen $\mathbb{D}^{1,2}$ and Vo Van Toi $\mathbb{D}^{1,2}$ \\ ${ }^{1}$ School of Biomedical Engineering, International University, Ho Chi Minh City 700000, Vietnam \\ ${ }^{2}$ Vietnam National University, Ho Chi Minh City 700000, Vietnam \\ Correspondence should be addressed to Phuoc Long Truong; tplong@hcmiu.edu.vn
}

Received 2 March 2021; Revised 27 April 2021; Accepted 16 May 2021; Published 2 June 2021

Academic Editor: Mai Duy Hien

Copyright (C) 2021 Phuoc Long Truong et al. This is an open access article distributed under the Creative Commons Attribution License, which permits unrestricted use, distribution, and reproduction in any medium, provided the original work is properly cited.

\begin{abstract}
Tebuconazole is a triazole fungicide used in agriculture to treat pathogenic fungi. It is listed as a possible carcinogen and it shows a potential risk for the environment at very low concentration. Therefore, the detection and monitoring of tebuconazole in food and environment play an important role. The current methods for the analysis of tebuconazole employ gas-liquid chromatography (GLC) and high-performance liquid chromatography (HPLC) after sample extraction with organic solvents and column cleaning. Besides the advantages of these methods such as efficiency, repeatability, and accuracy, they are still time-consuming and costly. Herein, we report a simple, sensitive platform for the fast detection of pesticides with a low cost. The detection technique exploits a pesticide-specific DNA aptamer as the bioreceptor of an optical biosensor. Instead of trying to capture the pesticide on the sensor surface, our method allows the DNA aptamers, which are adsorbed on the nanoparticle's surface, to detach from the nanoparticles when interacting with the pesticide. This leads to the pesticide-induced aggregation and the change of the absorption spectrum of metallic nanoparticles upon high-salt concentrations, which can be monitored with unaided eye or absorbance measurement. Using tebuconazole as a model analyte for detection of pesticide, the designed aptasensor showed a high sensitivity and selectivity with a detection limit of $\sim 10 \mathrm{nM}$ and reaction time within $\sim 20 \mathrm{~min}$. In the case of tebuconazole detection in spiked rice samples, the average recoveries were in the range of $89.90-110.86 \%$ with the relative standard deviations (RSD) of 3.11-4.32\%. These results indicate that our sensing platform can be exploited for the rapid detection of pesticides in real samples.
\end{abstract}

\section{Introduction}

Pesticides are crop protection products which play an important role in modern agriculture because of their ability to protect seeds and crops against insects, fungi, weeds, and other pests. Besides preventing crop loss and enhancing productivity, pesticides are also potentially toxic to humans $[1,2]$. They may cause environmental pollution and severe impairment of human health including cancer disease, effects on reproduction, and immune and nervous systems. The increasing globalization of agri-food production and consumption is now becoming more elongated. Food moves from country to country more frequently and in shorter time frames. As a result, contaminated foods have often been consumed before being approved by authorities. In order to minimize the health hazards caused by pesticides, governments and international organizations try to regulate the maximum residues level in agri-products and foodstuffs $[3,4]$.

Tebuconazole is a fungicide of the triazole group which treats pathogenic fungi on a plant by inhibiting ergosterol biosynthesis, an important component of fungal cell membranes, resulted in inhibition of growth and spore formation [5]. It is used in a wide range of crops such as cereals, nuts, grapes, and vegetables, and it is proved to be effective against powdery mildew, loose smuts, rusts of legume, and nonlegume crops. In soil, tebuconazole has a half-life ranging from 49 to 610 days under aerobic conditions [6]. Regular use of tebuconazole in agriculture leads to its accumulation in soils and, subsequently, can cause risks for surface water, groundwater, and soil ecosystems [7]. According to the United 
States Environmental Protection Agency (US EPA), tebuconazole is listed as a possible carcinogen with a rating of $\mathrm{C}$, and it has the potential to affect the endocrine system of different species by interacting with the steroidogenesis pathway [8].

Pesticide residues are a great concern for the public, and the monitoring of these chemical residues in water and foodstuffs is one of the most important steps in minimizing potential hazards to consumers. The standard methods for detection of pesticide residues are performed by various techniques such as gas-liquid chromatography (GLC), gas chromatography-mass spectrometry (GC-MS), and highperformance liquid chromatography (HPLC) after sample extraction with organic solvents and column cleaning [9]. Other methods such as immunoassays, electrochemical assays, and capillary gas chromatography are widely used for fast detection. These methods are sensitive and reliable, but each of them still has its shortcomings for food safety control such as complexity, high cost, time consumption including prerequisite sample pretreatment with the toxic organic reagents, inability for on-site testing, solution instability, and short storage time for testing $[10,11]$. For these reasons, there is now a great need of cost-efficient, rapid, and accurate detection methodologies.

In recent years, the progress in the field of nanomaterials has been made it possible to develop new sensors to overcome the disadvantages of the previous methods [11]. Among metallic nanoparticles, gold and silver nanoparticles (AuNPs and AgNPs) are of great interest due to their intrinsically strong surface plasmon resonance (SPR) absorptions, distance-dependent optical properties, high extinction coefficients, easy synthesis, and good biocompatibility [12]. The unique properties of noble metallic nanoparticles make them ideal probes for colorimetric assays to probe target analytes with the naked eye or with low-cost portable apparatus [13, 14]. The colorimetric assays have been developed by exploiting the color changes associated with the aggregation of nanoparticles, and they have been proved to be very useful in the determination of various analytes due to their outstanding analytical performances such as simplicity, sensitivity, specificity, rapidity, and on-site analysis with minimal reagent volumes. The sensing mechanism is based on the peak shift of absorption spectra of metallic nanoparticles due to the analyte-induced aggregation of the nanoparticles via the various interactions $[15,16]$. For sensing applications, metallic nanoparticles are usually required to conjugate with thiolated receptors. However, the immobilization is a timeconsuming process, and it is needed to optimize the amount of receptors loaded on the nanoparticle surface before sensing applications. In the case of AgNPs, the surface functionalization process that is time-consuming usually leads to the chemical degradation and oxidization of metal surface [12]. Recently, the electrostatic interaction between nonthiolated DNA and noble metallic nanoparticles has been exploited for biosensing $[17,18]$. In this case, DNA can be loaded on the nanoparticles in a short time at neutral $\mathrm{pH}$. The advent of aptamers, which are short, single-stranded DNA or RNA (ssDNA or ssRNA) molecules, called "artificial antibodies" has enabled the study of metallic nanoparticles for colorimet- ric aptasensors $[12,19]$. The aptamers loading on the nanoparticle surface make colorimetric assays more sensitive because the aggregation of nanoparticles is prevented in a high concentration of salt [20]. Currently, AuNPs are often used as sensing elements to develop colorimetric biosensors due to their chemical stability. As colorimetric assays, AgNPs are favored over AuNPs because they are cheaper and easier to synthesize and have higher extinction coefficients relative to AuNPs of the same size. Moreover, AgNPs have a stronger surface-enhanced Raman scattering (SERS) effect and surface plasmon resonance (SPR) absorption [21, 22]. Up to now, there are very few reports that described the use of AgNPs as colorimetric probes for the detection of fungicide. Therefore, the development of a simple colorimetric and spectrophotometric method with silver nanoparticles for rapid and on-site detection of tebuconazole has a great significance.

Herein, we report a simple, sensitive platform for the fast detection of pesticides with a low cost. The detection method exploits a pesticide-specific DNA aptamer as the recognition element of optical nanosensors. Instead of trying to capture pesticide on the sensor surface, our method allows the ssDNA aptamers, which are adsorbed on the silver nanoparticle's surface, to detach from the silver nanoparticles when interacting with pesticide. This leads to pesticide-induced aggregation of the silver nanoparticle upon high-salt concentrations, which can be monitored with unaided eye or simple absorbance measurement. Using tebuconazole as a model analyte for the detection of pesticides, the aptasensor could detect the tebuconazole with a detection limit as low as $10 \mathrm{nM}$ and the reaction time within $20 \mathrm{~min}$. With regard to demonstrating the sensitivity and specificity of the aptasensor, this sensing platform shows a great potential application for the fast and on-site detection of pesticides in real samples.

\section{Materials and Methods}

2.1. Chemicals and Materials. Silver nitrate $\left(\mathrm{AgNO}_{3}, 99 \%\right)$, Tebuconazole (99\%), sodium citrate dihydrate $\left(\mathrm{Na}_{3} \mathrm{C}_{6} \mathrm{H}_{5} \mathrm{O}_{7} \cdot 2 \mathrm{H}_{2} \mathrm{O}\right.$ ), phosphate buffer saline pH7.4 (PBS buffer), and sodium borohydride $\left(\mathrm{NaBH}_{4}, 98 \%\right)$ were obtained from Sigma-Aldrich, Inc. (USA). Acetone, ethanol, methanol, sodium chloride, nitric acid, and hydrochloric acid were purchased from Xilong Scientific Co. (China). The ssDNA aptamer of tebuconazole ( $5^{\prime}$ CGTACGGAATTCGC TAGCAGCGTCCACGAGTGTGGTGTGGAT CCGAGC TCCACGTG-3' ${ }^{\prime}$ [19] was obtained from PHUSA Biochem Co. (Vietnam). Other chemicals and essential reagents used were of AR grade. All glassware was intensively cleaned with freshly prepared aqua regia $\left(3: 1 \mathrm{HCl} / \mathrm{HNO}_{3}\right)$ and rinsed thoroughly with ultrapure water before use. Ultrapure water was used to prepare all chemical solutions.

\subsection{Synthesis and Characterization of Silver Nanoparticles.} Colloidal solution of citrate-capped silver nanoparticles (AgNPs) was prepared by reducing an aqueous solution of silver nitrate with a mixture of two reducing agents, trisodium citrate and sodium borohydride [23, 24]. In brief, $1 \mathrm{~mL}$ of $100 \mathrm{mM} \mathrm{AgNO}_{3}$ is added drop-wise to $99 \mathrm{~mL}$ of a vigorously stirred ice-cold solution containing $1 \mathrm{mM} \mathrm{NaBH}_{4}$ 
and $0.30 \mathrm{mM}$ trisodium citrate. The resulting solution was centrifuged for $15 \mathrm{~min}$ at $14000 \mathrm{rpm}$ in order to remove the excess of $\mathrm{NaBH}_{4}$, and the precipitation was redispersed in $2 \mathrm{mM}$ trisodium citrate. Then, the colloidal solution was filtered by a $0.22 \mu \mathrm{m}$ filter to eliminate aggregated nanoparticles. The morphology of citrate-capped AgNPs was characterized by UV-Vis spectrophotometer (Jasco V-730, Japan) and transmission electron microscopy (TEM; JEM1400, USA).

2.3. $p H$ Effect on the Stability of Silver Nanoparticles. $\mathrm{pH}$ is a key factor that affects the stability of nanoparticles. Hence, in order to assure that the nanoparticle aggregation is caused by the presence of the target analyte, we investigated the effect of $\mathrm{pH}$ on the stability of the silver colloidal solution. Briefly, the colloidal solution was centrifuged for $15 \mathrm{~min}$ at $14000 \mathrm{rpm}$, and the precipitation was redispersed in the solution with the $\mathrm{pH}$ in the range from 3 to 12 . The stability of colloidal nanoparticles was checked by the naked eye and UV-Vis spectrophotometer.

2.4. Effect of Solvents on Silver Nanoparticles. Solvents were used to stabilize silver nanoparticles and dissolve reactants as well as analytes. To determine the stability of silver nanoparticles with respect to solvents, the silver nanoparticles were redispersed in distilled water, PBS buffer ( $\mathrm{pH} 7.4)$, and organic solvents such as trisodium citrate $(2 \mathrm{mM})$, methanol, ethanol, and acetone. The stability of colloidal nanoparticles was recorded by the naked eye and UV-Vis spectrophotometer after 1-hour incubation.

2.5. Optimal Concentration of ssDNA Aptamer for Detection of Tebuconazole. Our sensing is platform based on the principle that silver nanoparticles and pesticides bind competitively to ssDNA aptamer. The ssDNA aptamer was absorbed on the nanoparticle's surface, and it stabilizes the nanoparticles against aggregation when adding salt with high concentration [19]. Its concentration directly influences on the sensitivity of the colorimetric assay. Therefore, it is needed to determine an optimal amount of DNA aptamer for the bioassay. In this study, ssDNA aptamer-wrapped silver nanoparticles were prepared based on the previous studies with modification $[25,26]$. Firstly, ssDNA aptamer was fully dehybridized and stretched by heating to $95^{\circ} \mathrm{C}$ for $2 \mathrm{~min}$. Then, ssDNA aptamer was diluted into various concentrations $(0,5,10,20,30,40,50,60$, and $70 \mathrm{pmol} / \mu \mathrm{L})$. Next, $7 \mu \mathrm{L}$ of different concentrations of ssDNA aptamer was thoroughly mixed with $350 \mu \mathrm{L}$ AgNPs and incubated for $30 \mathrm{~min}$ at room temperature following the addition of $350 \mu \mathrm{L}$ PBS pH 7.4. Subsequently, $35 \mu \mathrm{L}$ of $1.7 \mathrm{M} \mathrm{NaCl}$ was slowly added and mixed with the above solution and incubating for $10 \mathrm{~min}$. The stability of ssDNA aptamer-wrapped AgNPs was observed by the unaided eye and UV-Vis spectrophotometer. The minimum concentration of ssDNA aptamer that can stabilize the colloidal solution of silver nanoparticles was selected for biosensing.

2.6. Assay for Detection of Tebuconazole. $350 \mu \mathrm{L}$ of DNA aptamer wrapped AgNPs was mixed with $350 \mu \mathrm{L}$ of various concentrations of tebuconazole $(0,25,50,100,150,250$,

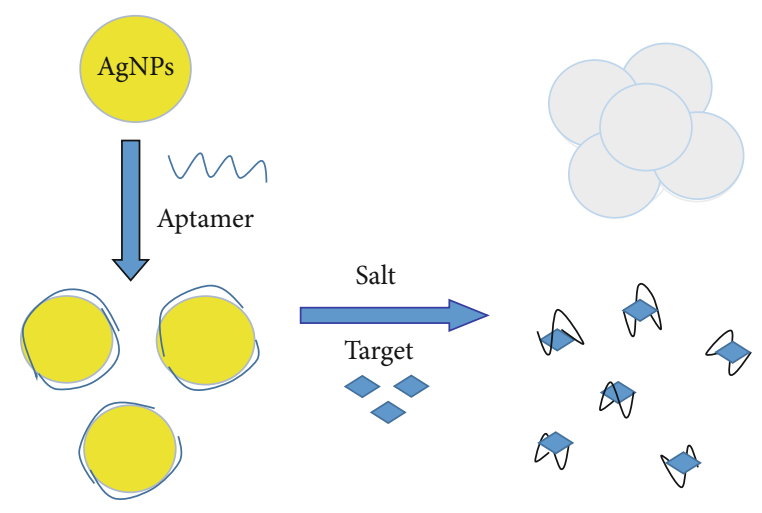

FIgURE 1: Experimental scheme depicting the experimental processes.

500 , and $750 \mathrm{nM}$ ) and incubated for $10 \mathrm{~min}$ at room temperature. After adding $35 \mu \mathrm{L}$ of $1.7 \mathrm{M} \mathrm{NaCl}$ into the incubated mixture, the color change and spectra response of DNA aptamer wrapped AgNPs with tebuconazole were observed by the naked eye and/or an UV-Vis spectrophotometer. To ensure the specificity of the DNA aptamer, a series of other pesticides were examined under the same procedure. These pesticides were as follows: difenoconazole, hexaconazole, thiophanate-methyl, mancozeb, azoxystrobin, and carbendazim.

2.7. Application to Spiked Rice Samples. To assess the feasibility of sensing platform in real samples, the sensing platform was applied to detect tebuconazole in the spiked rice samples. The spiked rice samples were prepared based on the previous report with minor modification [27]. In brief, $2 \mathrm{~g}$ of rice flour was spiked with various concentrations of tebuconazole (50, 100 , and $250 \mathrm{nM}$ ), respectively, and incubated at room temperature for $30 \mathrm{~min}$ for better absorbance of the pesticide in the matrix. Next, the spiked samples were mixed with $4 \mathrm{~mL}$ of acetonitrile and $1 \mathrm{~mL}$ distilled water, followed by ultrasonic extraction for $30 \mathrm{~min}$ and centrifugation at $5000 \mathrm{rpm}$ for $15 \mathrm{~min}$. Then, the supernatant was collected and evaporated at $40^{\circ} \mathrm{C}$. The residue was diluted with water for further analysis. Then, the tebuconazole was detected using the aptasensor above.

\section{Results and Discussion}

The sensing mechanism of the proposed platform is based on the adsorption of the single-stranded DNA (ssDNA) on the metallic nanoparticles in a colloidal solution [28]. ssDNA is capable to uncoil its bases to make them exposed to the nanoparticle's surface. The electrostatic interaction between the positively charged bases of ssDNA and the negative charge of nanoparticles helps attach the ssDNA on the nanoparticle surface. Therefore, ssDNA can stabilize the nanoparticle against aggregation via electrostatic repulsion when adding salt with high concentration [25]. In this study, we used DNA aptamer as a specific recognition element and bare AgNPs as a signal transducer element. As illustrated in Figure 1, our detection method is based on the principle that 


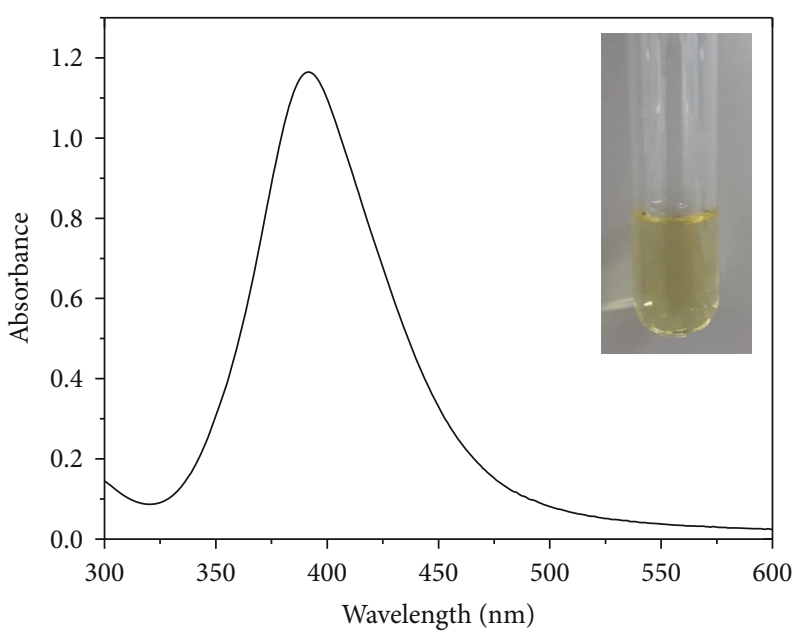

(a)

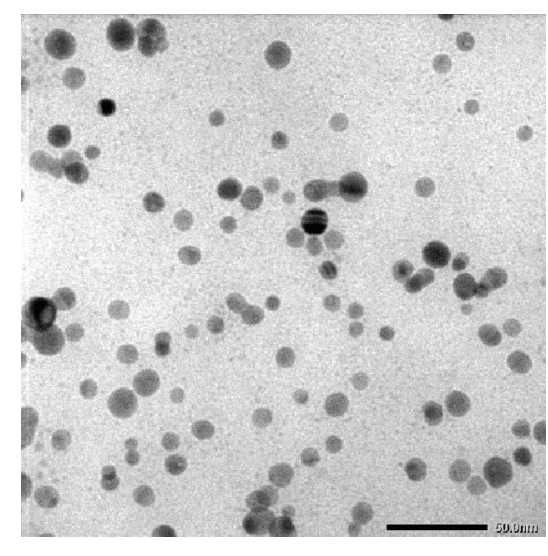

(b)

FIGURE 2: (a) UV-Vis absorption spectrum of Ag nanoparticles. (b) Size and morphology of Ag nanoparticles with a diameter of $\sim 12 \mathrm{~nm}$ (TEM).

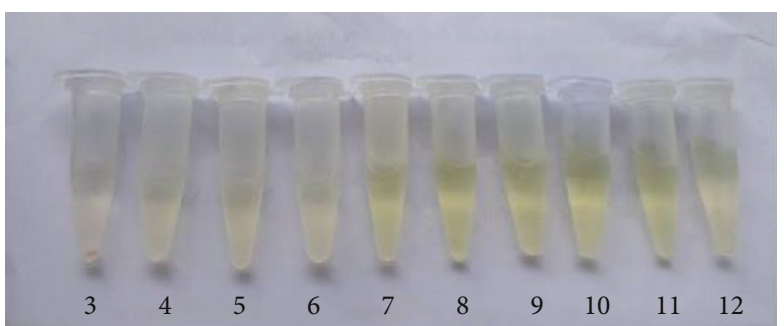

(a)

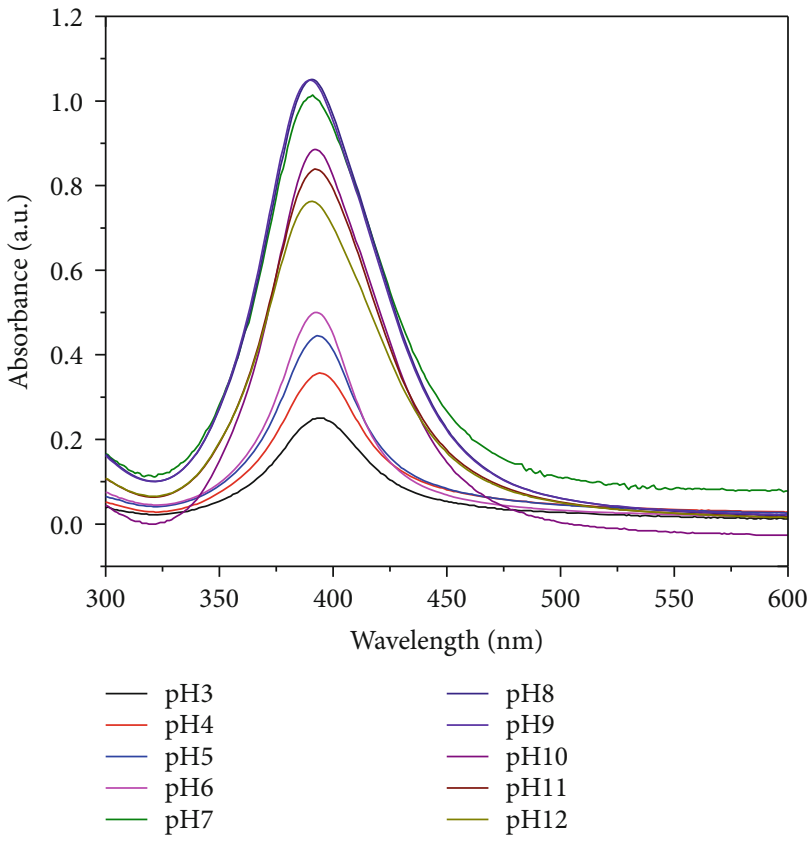

(b)

FIgURE 3: (a) The color of colloidal solution of silver nanoparticles in solvents with different pH values. (b) UV-Vis spectra of silver nanoparticles in solutions with different $\mathrm{pH}$ values.

target pesticide and AgNPs bind competitively to DNA aptamer. The DNA aptamer was absorbed on the nanoparticle's surface, and it stabilizes the nanoparticles against aggregation via the electrostatic repulsion when adding salt with high concentration [29]. The electrostatic repulsion helps prevent the strong van der Waals attraction and enhances the stability of metallic nanoparticles. The conformation of DNA aptamer changes from a randomly coiled structure to a folded 3D structure when adding target pesticide that made DNA aptamers detach from the nanoparticle sur- face. This transition resulted in the subsequent aggregation of the nanoparticles when adding a high concentration of salt. This leads to the changes of the UV-Vis absorption spectrum and the characteristic color change of colloidal silver.

The first step in this study involved the synthesis of the colloidal silver nanoparticles using the classical chemical synthesis method. The silver nitrate was reduced by sodium borohydride and trisodium citrate to form AgNPs, and the remaining citrate helps stabilize AgNPs. The reaction occurs 


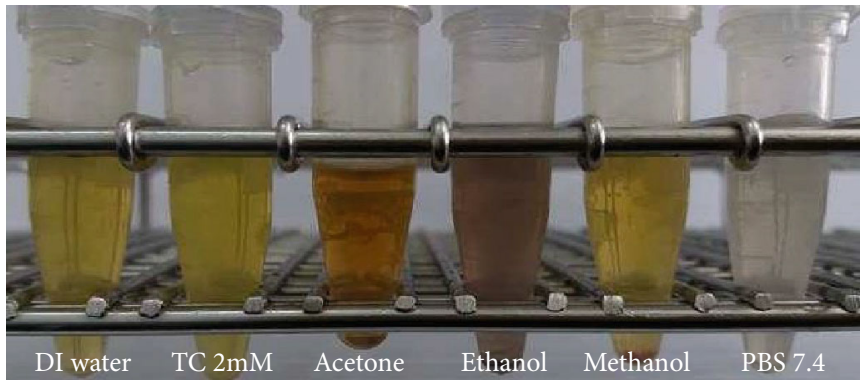

(a)

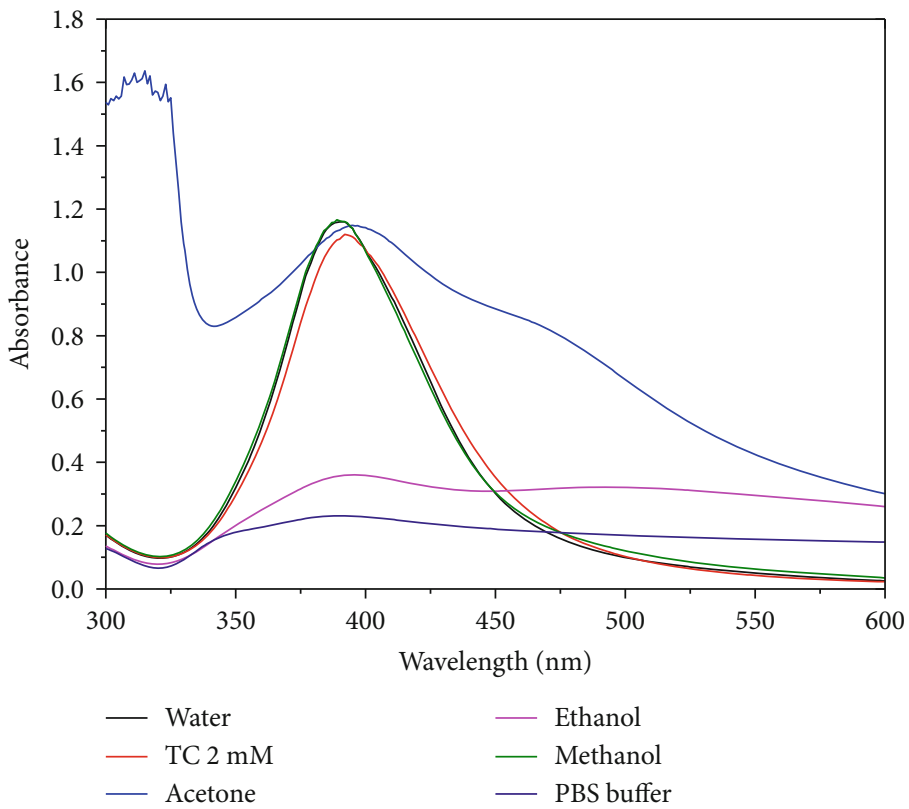

(b)

FIGURE 4: (a) The visual color changes of colloidal silver in various solvents. (b) UV-Vis spectra of silver nanoparticles in various solvents.

at room temperature with very fast rate $[23,30]$. This method yielded pale yellow colored aqueous dispersion of silver nanoparticles with a diameter of $\sim 12 \mathrm{~nm}$, as revealed by $\mathrm{UV}-\mathrm{V}$ is spectrophotometer and transmission electron microscopy (TEM). The silver nanoparticles were nearly spherical in shape and had a size dispersion of only a few nanometers, and the wavelength of surface plasmon resonance (SPR) occurred at $393 \mathrm{~nm}$ (Figure 2). The concentration of citrate-capped silver nanoparticles was $\sim 2.0 \mathrm{nM}$ calculated based on Beer-Lambert law and the average diameter of silver nanoparticles [24].

It is well-known that the applications of the silver nanoparticles in assays depend on their stability, especially for those based on the aggregation of noble metal nanoparticles [31]. Silver nanoparticles prepared in this research are relatively stable and well dispersed in solution because of the negative charge of the citrate layer on the nanoparticle surface. The electrostatic repulsions among neighboring nanoparticles due to negative surface charge keep them remain dispersed in solution. $\mathrm{pH}$ is one of the critical factors that affect the stabilization of the nanoparticles. Hence, we investigated the effect of $\mathrm{pH}$ in the range from 3 to 12 . Figure 3 shows the visual observation and UV-Vis spectra of colloidal solution of silver nanoparticles with different $\mathrm{pH}$ values. At $\mathrm{pH}<7$, the silver nanoparticles were unstable, and the color of nanosilver solutions was changed from yellow to yellowish indicating aggregation of the nanoparticles. These observations were supported by the UV-Vis spectra of colloidal silver nanoparticles. The UV-Vis spectrum showed a significant decrease in the absorbance at $393 \mathrm{~nm}$, and the absorbance further decreased when decreasing $\mathrm{pH}$ of the colloidal solution. This is because citrate is fully protonated at acidic $\mathrm{pH}$ values, and hence, the number of negative charge on the silver nanoparticle surface is reduced. The decrease in negative surface charges results in the aggregation of the nanoparticles. For the silver colloidal solution with the $\mathrm{pH}$ in the alkaline region, the decrease in the peak absorbance was much less than that of the acidic $\mathrm{pH}$ region. This indicated that the solution was more stabilized in the alkaline $\mathrm{pH}$ region. The reason is that the citrate is fully deprotonated, and there are more negative charges that result in repulsion among silver nanoparticles, and hence, little or no aggregation was observed. At a $\mathrm{pH}$ range of 7-9, there was almost no change in the peak absorbance that indicated no aggregation of the 


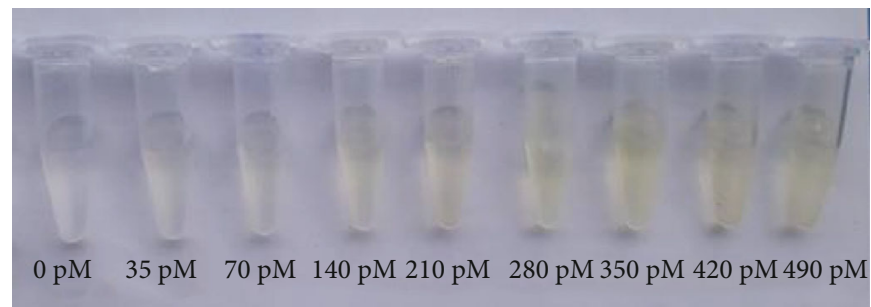

(a)

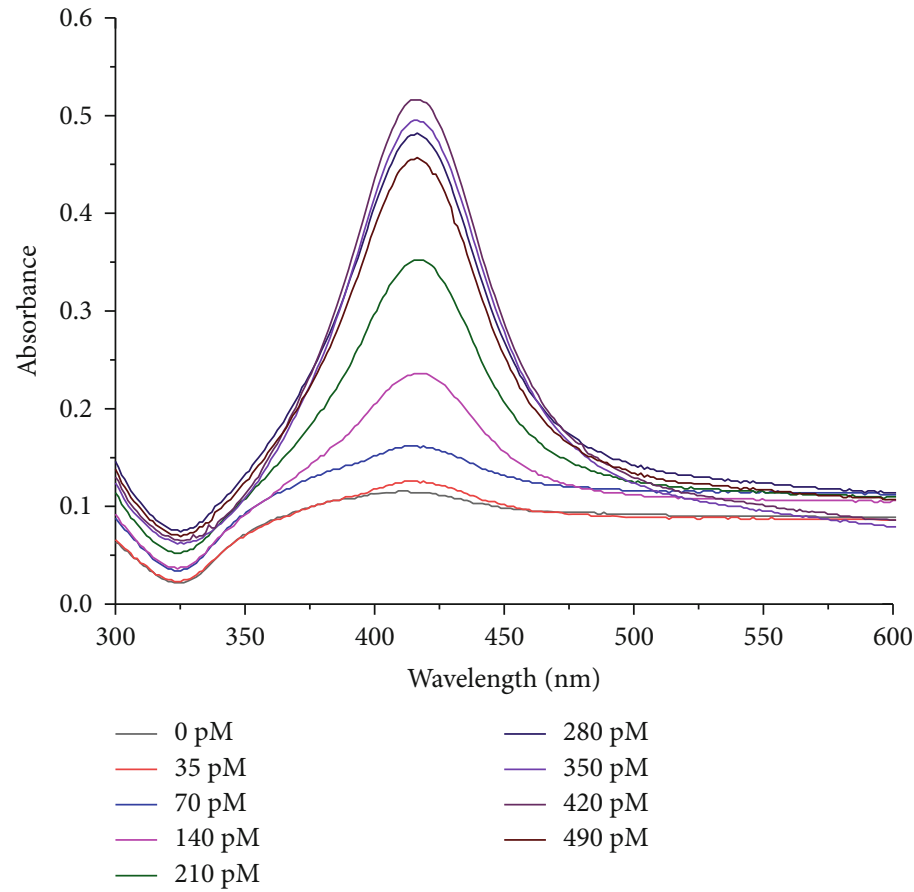

(b)

FIgURE 5: (a) The color change of colloidal solution of silver nanoparticles stabilized by different amounts of DNA aptamer. (b) UV-Vis spectra of colloidal silver nanoparticles stabilized by different amounts of DNA aptamer after salt addition.

silver nanoparticles. Therefore, this $\mathrm{pH}$ range was chosen for further studies.

Solvent is one of the most important factors that affect the stability of the colloidal solution. The changing of the dielectric environment of silver nanoparticles is very important as it relates to the ability to process metal nanoparticles in various solutions for applications [32]. Furthermore, solvents can have an effect on the solubility and stability of pesticides. To determine the effect of solvents on colloidal silver, the silver nanoparticles were centrifuged and redispersed in various solvents. The stability of silver nanoparticle was evaluated by the color change of the colloidal solution and UVVis spectrum after one-hour incubation. As demonstrated in Figure 4, three solvents (PBS pH 7.4, ethanol, and acetone) significantly turned the solution color and the UV-Vis spectrum of silver colloids that indicate the aggregation of silver nanoparticles. In the case of water, trisodium citrate, and methanol, the silver nanoparticles are nicely redispersed, and all 3 solvents had little to no effect on the solution color and the UV-Vis spectrum of silver nanoparticles. That is because these solvents provide solvation and driving forces that prevent the aggregation of silver nanoparticles. In this research, ultrapure water and sodium citrate solution with a concentration of $2 \mathrm{mM}$ were used as solvents to dilute the stock solutions of pesticides and disperse silver nanoparticles.

As mentioned above, DNA aptamer was used as a recognition factor and the AgNPs was used as a signal transducer element. Hence, the aptamer amount and the ratio of DNA aptamer and AgNPs in the assays directly affect the sensitivity of the sensor. It should be noted that the electrostatic interaction between AgNPs and the bases of DNA is a key factor to cause aptamer to stick on the nanoparticle's surface. For this reason, the DNA amount used for these assays directly relates to the length of DNA aptamer and its sequence. To identify an optimal ratio of DNA aptamer to AgNPs at which the aptamer-AgNPs remains stable in the absence of target pesticide after addition of high-level salt, different amounts of DNA aptamer were examined. As shown in Figure 5, DNA could protect the AgNPs from the aggregation in solutions with high salinity. In the presence of 280 pmol of DNA, the silver colloid could keep stable in a solution containing a high concentration of salt. With the 


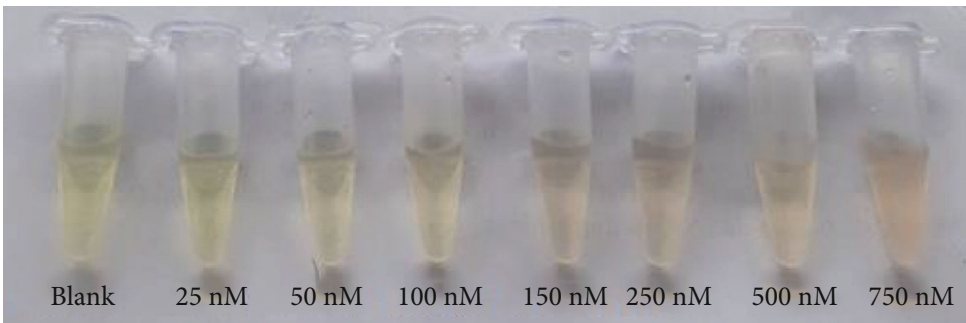

(a)

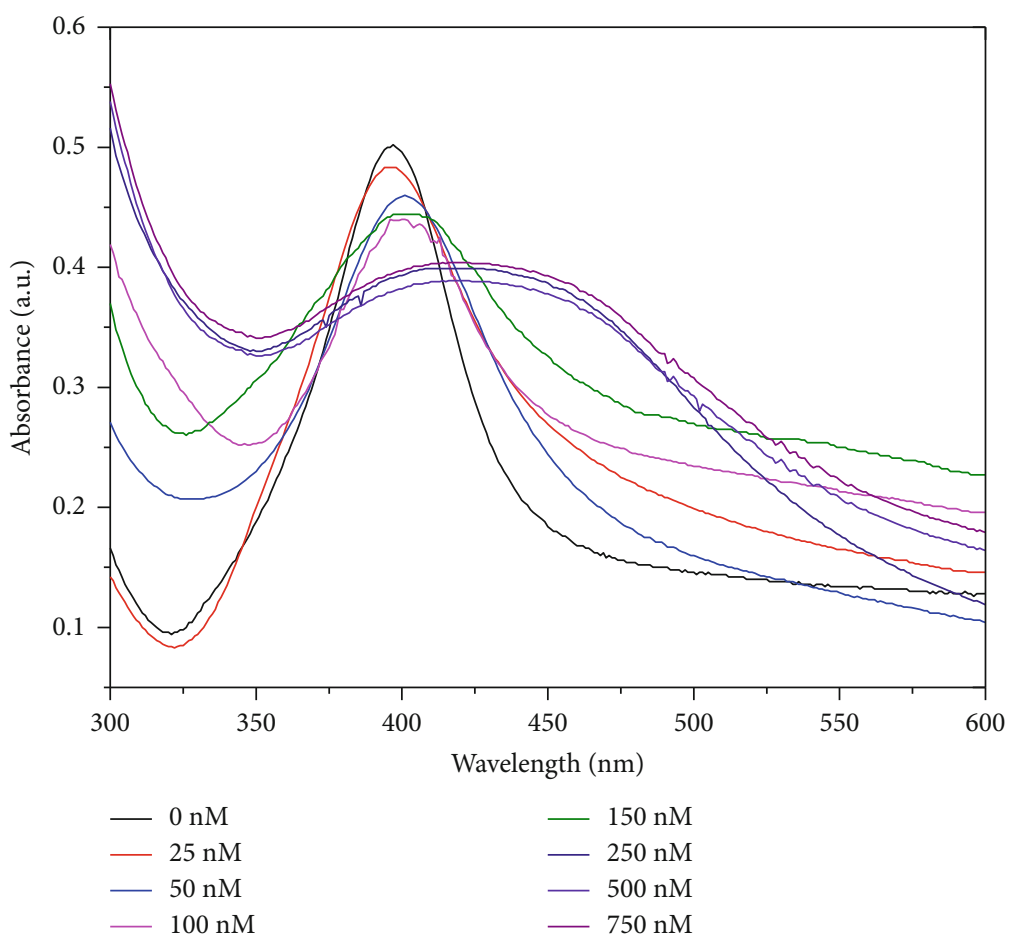

(b)

Figure 6: (a) Colorimetric response of DNA aptamer modified AgNPs as a function of tebuconazole concentration. (b) UV-Vis spectral response of aptasensor in the presence of various amounts of tebuconazole.

lower amount of DNA aptamer, the absorbance of AgNPs at $393 \mathrm{~nm}$ gradually decreased, and the color of silver solutions was changed from yellow to yellowish indicating aggregation of the nanoparticles. With the higher amount of DNA aptamer, the colloidal solutions of nanoparticles maintain its original yellow color, and the absorbance of AgNPs at $393 \mathrm{~nm}$ indicated no aggregation of the nanoparticles. The successful fabrication of the aptamer-wrapped AgNPs was confirmed by the measurement of the zeta potential of nanoparticles. The zeta potential of citrate-capped AgNPs was $-42.35 \pm 3.10 \mathrm{mV}(n=3)$ due to the presence of citrate on the nanoparticle surface. After modification, the zeta potential of the aptamer-wrapped AgNPs was $38.22 \pm 1.70 \mathrm{mV}(n=3)$. This indicates that the AgNPs were stabilized by ssDNA aptamer. Hence, the optimal ratio of DNA aptamer and AgNPs used for detection of tebuconazole was $280 \mathrm{pM} / 350 \mu \mathrm{L}$ AgNPs with a concentration of $\sim 2.0 \mathrm{nM}$.

After optimizing the reaction conditions, the sensitivity of the proposed DNA aptasensor was investigated. For pesti- cide analysis, different concentrations of tebuconazole ranging from 0 to $750 \mathrm{nM}$ were incubated with DNA aptamer wrapped AgNPs, and the color change and spectra response were recorded. As illustrated in Figure 6, in the presence of tebuconazole, DNA aptamers bind to tebuconazole and form a structured complex, resulting in the aggregation of AgNPs after salt addition that leads to the characteristic color change from yellow to pink (Figure 6(a)). With the increase of tebuconazole concentration, the UV-Vis spectra of AgNPs showed that the characteristic absorbance at $393 \mathrm{~nm}$ decreased, while the absorbance band $\sim 450 \mathrm{~nm}$ increased progressively which indicated the aggregation of AgNPs (Figure 6(b)). In this study, the absorbance ratio (A450/A393) was used to evaluate the tebuconazoleinduced aggregation level of DNA aptamer-wrapped AgNPs and the sensitivity of aptasensor. Figure 7(a) demonstrates the relationship between the absorbance ratio (A450/A393) and tebuconazole concentrations, and the ratio was found to be linear in the concentration range of $25 \mathrm{nM}$ to $250 \mathrm{nM}$. The linear regression equation for detection of tebuconazole 


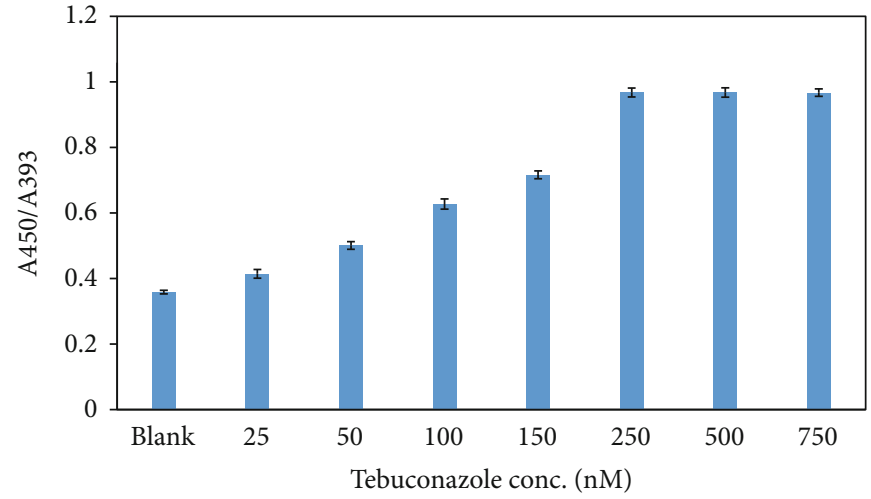

(a)

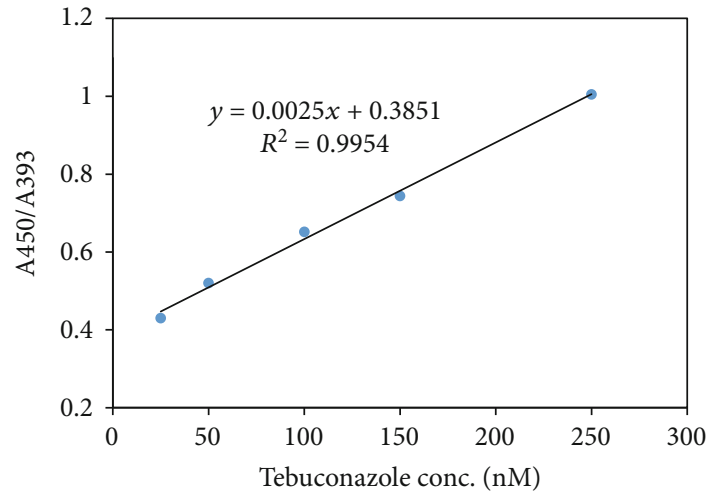

(b)

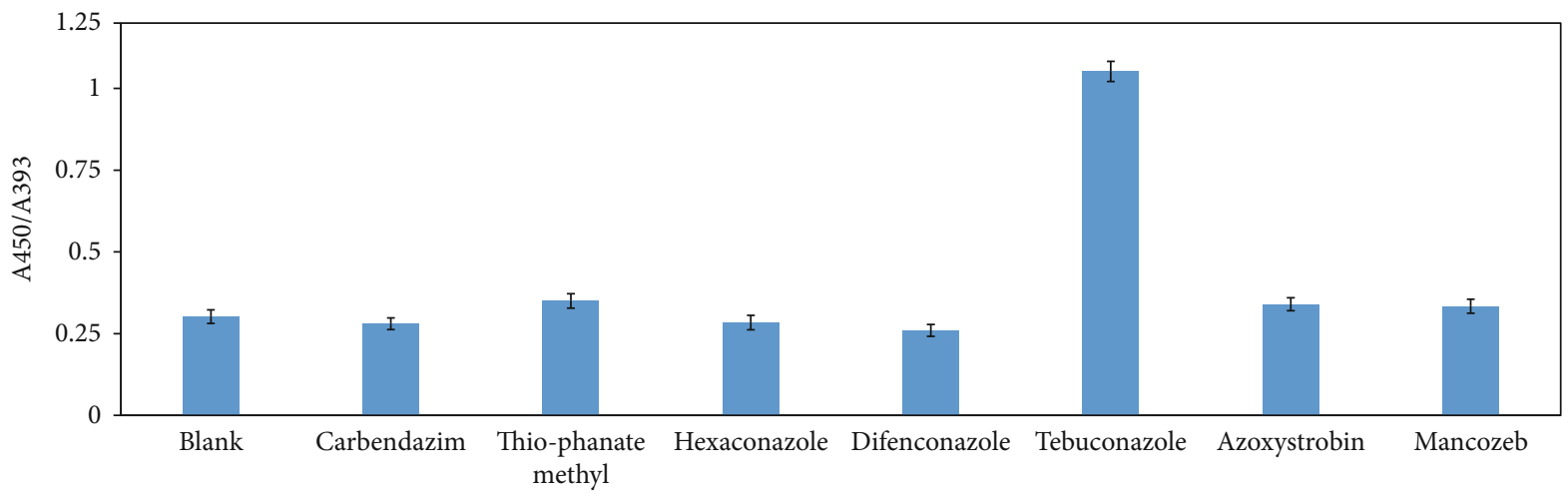

(c)

FIgURE 7: (a) The response of absorbance ratio (A450/A393) for the various concentrations of tebuconazole ranging from 0 to $750 \mathrm{nM}$. (b) The linear scale of the calibration curve describing the relation between absorbance ratio (A450/A393) and concentrations of tebuconazole. (c) Specificity of aptasensor in the presence of different pesticides.

was $y=0.0025 x+0.3851\left(R^{2}=0.9954\right)$, where $y$ and $x$ are the ratio of $\mathrm{A}_{450} / \mathrm{A}_{393}$ and tebuconazole concentration (nM), respectively (Figure $7(\mathrm{~b})$ ). The detection limit was calculated based on the formula $3 \alpha /$ s, where $\alpha$ is the standard deviation of the plank sample and $s$ is the slope of the linear scale of the calibration curve. Therefore, the detection limit of this aptasensor is $\sim 10 \mathrm{nM}$, which is about two orders of magnitude lower than the maximum residue limit (MRL) of tebuconazole in agri-products (Nos. EU 750/2010 and 50/2016/TT-BYT) [33, 34]. Moreover, in comparison with other assays for detection of tebuconazole that require expensive reagents, costly instrumentation, sample pretreatment with the toxic organic reagents such as immunoassay [35, 36], liquid chromatography (LC) with UV detection (LCUV) [37], and capillary gas chromatography with nitrogen phosphorus detection (GC-NPD) [38], this sensing platform shows its advantages in terms of simplicity, experimental cost, time for analysis, and ability for on-site testing.

To ensure the observed absorbance ratio of A450/A393 from the specific interaction of tebuconazole and aptamerAgNPs, the specificity of the aptasensor was investigated on a series of interfering pesticides such as difenoconazole, hexaconazole, thiophanate-methyl, mancozeb, azoxystrobin, and carbendazim at concentrations which were larger than the concentration of the saturation response of aptasensor.
TABle 1: Detection of tebuconazole in spiked rice samples.

\begin{tabular}{lccc}
\hline $\begin{array}{l}\text { Spiked concentration } \\
(\mathrm{nM})\end{array}$ & $\begin{array}{c}\text { Found concentration } \\
(\mathrm{nM})\end{array}$ & $\begin{array}{c}\text { Recovery } \\
(\%)\end{array}$ & $\begin{array}{c}\text { RSD } \\
(\%)\end{array}$ \\
\hline 50 & 55.43 & 110.86 & 3.65 \\
100 & 89.90 & 89.9 & 3.11 \\
250 & 248.40 & 99.36 & 4.32 \\
\hline
\end{tabular}

As shown in Figure $7(\mathrm{c})$, the absorbance ratio of A450/A393 in the presence of tebuconazole was much larger than those of other pesticides. This result indicated that this sensing platform could be used for the detection of pesticides with high specificity.

To evaluate the efficacy of the proposed aptasensor for the analysis of complex samples, spiked rice samples were tested. As demonstrated in Table 1, the average recoveries were in the range of $89.90-110.86 \%$; the relative standard deviations (RSD) were 3.11-4.32\%. The recovery results are in good agreement with those obtained from gasliquid chromatography (GLC) and liquid chromatography-mass spectrometry. These results confirmed the reliability and practicability of the proposed sensing platform for the simple and fast detection of tebuconazole in real samples as well. 


\section{Conclusions}

In summary, we developed a simple aptasensor for the fast detection of tebuconazole fungicide at a low cost based on the aggregation of AgNPs. This approach is suitable for onsite detection because the aggregation of the nanoparticle is very fast and easy to visualize through the color and state of the resultant solution. Using tebuconazole as a model for the detection of pesticides, the plasmonic aptasensor could detect as low as $10 \mathrm{nM}$ with a reaction time of $\sim 20 \mathrm{~min}$ and the plotted calibration curve in the concentration range of 25 to $250 \mathrm{nM}$. Moreover, the tebuconazole was successfully detected in spiked rice samples with the average recoveries ranging from 89.90 to $110.86 \%$ and the relative standard deviations (RSD) of $3.11-4.32 \%$. The above results show that the proposed aptasensor can detect tebuconazole with high sensitivity and selectivity. In addition, the tebuconazole induced color change of aptamer-modified AgNPs from yellow to pink enables visual detection of tebuconazole in real applications.

\section{Data Availability}

All data used to support the findings of this study are included within the article.

\section{Conflicts of Interest}

The authors have no conflicts of interest.

\section{Acknowledgments}

This research is funded by the Vietnam National University Ho Chi Minh City (VNU-HCM) under grant number NCM2020-28-01.

\section{References}

[1] S. Armenta, G. Quintas, S. Garrigues, and M. De la Guardia, "Mid-infrared and Raman spectrometry for quality control of pesticide formulations," TrAC Trends in Analytical Chemistry, vol. 24, no. 8, pp. 772-781, 2005.

[2] A. Nougadère, J. C. Reninger, J. L. Volatier, and J. C. Leblanc, "Chronic dietary risk characterization for pesticide residues: a ranking and scoring method integrating agricultural uses and food contamination data," Food and Chemical Toxicology, vol. 49, no. 7, pp. 1484-1510, 2011.

[3] A. Pop, F. Manea, A. Flueras, and J. Schoonman, "Simultaneous voltammetric detection of carbaryl and paraquat pesticides on graphene-modified boron-doped diamond electrode," Sensors, vol. 17, no. 9, p. 2033, 2017.

[4] X. Liu, X. Wang, J. Xu, F. Dong, W. Song, and Y. Zheng, "Determination of tebuconazole, trifloxystrobin and its metabolite in fruit and vegetables by a Quick, Easy, Cheap, Effective, Rugged and Safe (QuEChERS) method using gas chromatography with a nitrogen-phosphorus detector and ion trap mass spectrometry," Biomedical Chromatography, vol. 25, no. 10, pp. 1081-1090, 2011.

[5] N. T. Sehnem, P. Souza-Cruz, M. D. C. R. Peralba, and M. A. Z. Ayub, "Biodegradation of tebuconazole by bacteria isolated from contaminated soils," Journal of Environmental Science and Health Part B, vol. 45, no. 1, pp. 67-72, 2009.

[6] B. Muñoz-Leoz, E. Ruiz-Romera, I. Antigüedad, and C. Garbisu, "Tebuconazole application decreases soil microbial biomass and activity," Soil Biology and Biochemistry, vol. 43, no. 10, pp. 2176-2183, 2011.

[7] R. Norková, J. J. Dytrtová, M. Jakl, and D. Schröder, "Formation of tebuconazole complexes with cadmium (II) investigated by electrospray ionization mass spectrometry," Water, Air, \& Soil Pollution, vol. 223, no. 5, pp. 2633-2640, 2012.

[8] R. Noguerol-Pato, R. M. González-Rodríguez, C. González-Barreiro, B. Cancho-Grande, and J. Simal-Gándara, "Influence of tebuconazole residues on the aroma composition of Mencía red wines," Food Chemistry, vol. 124, no. 4, pp. 1525-1532, 2011.

[9] M. L. Xu, Y. Gao, X. X. Han, and B. Zhao, "Detection of pesticide residues in food using surface-enhanced Raman spectroscopy: a review," Journal of Agricultural and Food Chemistry, vol. 65, no. 32, pp. 6719-6726, 2017.

[10] E. Watanabe, S. Miyake, and Y. Yogo, "Review of enzymelinked immunosorbent assays (ELISAs) for analyses of neonicotinoid insecticides in agro-environments," Journal of Agricultural and Food Chemistry, vol. 61, no. 51, pp. 1245912472, 2013.

[11] G. Aragay, F. Pino, and A. Merkoçi, "Nanomaterials for sensing and destroying pesticides," Chemical Reviews, vol. 112, no. 10, pp. 5317-5338, 2012.

[12] M. Sabela, S. Balme, M. Bechelany, J.-M. Janot, and K. Bisetty, "A review of gold and silver nanoparticle-based colorimetric sensing assays," Advanced Engineering Materials, vol. 19, 2017.

[13] J. V. Rohit and S. K. Kailasa, "5-Sulfo anthranilic acid dithiocarbamate functionalized silver nanoparticles as a colorimetric probe for the simple and selective detection of tricyclazole fungicide in rice samples," Analytical Methods, vol. 6, no. 15, pp. 5934-5941, 2014.

[14] J.-S. Lee, A. K. R. Lytton-Jean, S. J. Hurst, and C. A. Mirkin, "Silver nanoparticle- oligonucleotide conjugates based on DNA with triple cyclic disulfide moieties," Nano Letters, vol. 7, no. 7, pp. 2112-2115, 2007.

[15] H. Jans and Q. Huo, "Gold nanoparticle-enabled biological and chemical detection and analysis," Chemical Society Reviews, vol. 41, no. 7, pp. 2849-2866, 2012.

[16] R. Elghanian, J. J. Storhoff, R. C. Mucic, R. L. Letsinger, and C. A. Mirkin, "Selective colorimetric detection of polynucleotides based on the distance-dependent optical properties of gold nanoparticles," Science, vol. 277, no. 5329, pp. 1078-1081, 1997.

[17] S. Abbasian, A. Moshaii, M. Nikkhah, and N. Farkhari, "Adsorption of DNA on colloidal Ag nanoparticles: effects of nanoparticle surface charge, base content and length of DNA," Colloids and Surfaces B: Biointerfaces, vol. 116, pp. 439-445, 2014.

[18] N. Farkhari, S. Abbasian, A. Moshaii, and M. Nikkhah, "Mechanism of adsorption of single and double stranded DNA on gold and silver nanoparticles: investigating some important parameters in bio-sensing applications," Colloids and Surfaces B: Biointerfaces, vol. 148, pp. 657-664, 2016.

[19] V. T. Nguyen, Y. S. Kwon, J. H. Kim, and M. B. Gu, "Multiple GO-SELEX for efficient screening of flexible aptamers," Chemical Communications, vol. 50, no. 72, pp. 10513-10516, 2014.

[20] J. Wang, L. Wang, X. Liu et al., "A gold nanoparticle based aptamer target binding readout for ATP assay," Advanced Materials, vol. 19, no. 22, pp. 3943-3946, 2007. 
[21] X. Xu, J. Wang, F. Yang, K. Jiao, and X. Yang, "Label-free colorimetric detection of small molecules utilizing DNA oligonucleotides and silver nanoparticles," Small, vol. 5, no. 23, pp. 2669-2672, 2009.

[22] S. K. Menon, N. R. Modi, A. Pandya, and A. Lodha, "Ultrasensitive and specific detection of dimethoate using apsulphonato-calix [4] resorcinarene functionalized silver nanoprobe in aqueous solution," RSC Advances, vol. 3, no. 27, pp. 10623-10627, 2013.

[23] G. L. Wang, X. Y. Zhu, H. J. Jiao, Y. M. Dong, and Z. J. Li, "Ultrasensitive and dual functional colorimetric sensors for mercury (II) ions and hydrogen peroxide based on catalytic reduction property of silver nanoparticles," Biosensors and Bioelectronics, vol. 31, no. 1, pp. 337-342, 2012.

[24] D. Paramelle, A. Sadovoy, S. Gorelik, P. Free, J. Hobley, and D. G. Fernig, "A rapid method to estimate the concentration of citrate capped silver nanoparticles from UV-visible light spectra," Analyst, vol. 139, no. 19, pp. 4855-4861, 2014.

[25] M. Ramezani, N. M. Danesh, P. Lavaee, K. Abnous, and S. M. Taghdisi, "A novel colorimetric triple-helix molecular switch aptasensor for ultrasensitive detection of tetracycline," Biosensors and Bioelectronics, vol. 70, pp. 181-187, 2015.

[26] W. H. Wu, M. Li, Y. Wang et al., "Aptasensors for rapid detection of Escherichia coli O157: H7 and Salmonella typhimurium,” Nanoscale Research Letters, vol. 7, no. 1, p. 658, 2012.

[27] N. Fahimi-Kashani and M. R. Hormozi-Nezhad, "Gold-nanoparticle-based colorimetric sensor array for discrimination of organophosphate pesticides," Analytical Chemistry, vol. 88, no. 16, pp. 8099-8106, 2016.

[28] H. Li and L. Rothberg, "Colorimetric detection of DNA sequences based on electrostatic interactions with unmodified gold nanoparticles," Proceedings of the National Academy of Sciences, vol. 101, no. 39, pp. 14036-14039, 2004.

[29] Y. Wang, J. Wang, F. Yang, and X. Yang, "Spectrophotometric determination of cysteine with gold nanoparticles stabilized with single-stranded oligonucleotides," Analytical Sciences, vol. 26, no. 5, pp. 545-549, 2010.

[30] S. Preciado-Flores, D. A. Wheeler, T. M. Tran et al., "SERS spectroscopy and SERS imaging of Shewanella oneidensis using silver nanoparticles and nanowires," Chemical Communications, vol. 47, no. 14, pp. 4129-4131, 2011.

[31] R. C. Doty, T. R. Tshikhudo, M. Brust, and D. G. Fernig, "Extremely stable water-soluble Ag nanoparticles," Chemistry of Materials, vol. 17, no. 18, pp. 4630-4635, 2005.

[32] M. A. H. Muhammed, F. Aldeek, G. Palui, L. TrapiellaAlfonso, and H. Mattoussi, "Growth of in situ functionalized luminescent silver nanoclusters by direct reduction and size focusing," ACS Nano, vol. 6, no. 10, pp. 8950-8961, 2012.

[33] "EU pesticide database," https://eurlex.europa.eu/ legalcontent/EN/ALL/?uri=CELEX:32010R0750.

[34] "Vietnam. Maximum residue limits for pesticides in food. 50/2016/TT-BYT," https://www.fas.usda.gov/data/vietnammoh-revises-mrls-pesticides-foods.

[35] Y. Wang, J. Xu, Y. Qiu et al., "Highly specific monoclonal antibody and sensitive quantum dot beads-based fluorescence immunochromatographic test strip for tebuconazole assay in agricultural products," Journal of Agricultural and Food Chemistry, vol. 67, no. 32, pp. 9096-9103, 2019.

[36] X. Chen, Z. Li, F. Sun et al., "An innovative hapten and monoclonal antibody-based immunoassay for determining tebuco- nazole residues in aqueous samples," Food and Agricultural Immunology, vol. 30, no. 1, pp. 677-691, 2019.

[37] T. Miyauchi, M. Mori, and K. Ito, "Application of solid-phase extraction to quantitatively determine cyproconazole and tebuconazole in treated wood using liquid chromatography with UV detection," Journal of Chromatography A, vol. 1063, no. 1-2, pp. 137-141, 2005.

[38] Z. Deng, J. Hu, D. Qin, and H. Li, "Simultaneous analysis of hexaconazole, myclobutanil, and tebuconazole residues in apples and soil by SPE clean-up and GC with nitrogen-phosphorus detection," Chromatographia, vol. 71, no. 7-8, pp. 679-684, 2010. 